\title{
Performance of human papillomavirus DNA detection in residual specimens taken for Chlamydia trachomatis and Neisseria gonorrhoeae nucleic acid amplification testing in men who have sex with men
}

Diarmuid Nugent (1), Oliver Stirrup (1), Sarah Pett (1,2), Kavita Panwar (3), Marta Checchi (3), David Mesher (3), Kate Soldan (3), Simon Beddows (3), Richard Gilson (1,2)

1. UCL Centre for Clinical Research in Infection and Sexual Health, Institute for Global Health, University College London, London WC1E 6JB; 2. The Mortimer Market Centre, Central and North West London NHS Foundation Trust, London WC1E 6JB; 3. Public Health England, London NW9 5EQ

\section{Key Points}

- Anal HPV infection was highly prevalent in our sample of men who have sex with men (MSM) attending a London sexual health service

- HPV detection from residual rectal and pooled STI test specimens in MSM is highly concordant with a dedicated anal swab specimen

- This study supports using residual STI test specimens for surveillance of HPV in MSM, including to evaluate the impact of vaccination programmes

- If sexual health services increase their use of pooled STI test specimens in the future, this should have little impact on estimates of HPV prevalence

\footnotetext{
Abstract

Word count $=300$

Objectives

Rectal swab specimens, either alone, or pooled with first void urine (FVU) and pharyngeal swab specimens, are used to test for Chlamydia trachomatis (CT) and Neisseria gonorrhoeae (NG) infection in men who have sex with men (MSM). Following introduction of human papillomavirus (HPV) vaccination for MSM attending UK sexual health services (SHS), HPV testing of residual CT/NG test specimens has been proposed to monitor HPV prevalence in this population. Performance of HPV detection in such specimens has not been evaluated previously.
} 
Methods

MSM attending a UK SHS provided 3 specimens: (i) rectal swab for CT/NG; (ii) pooled rectal/pharyngeal/FVU specimen for CT/NG ; (iii) dedicated anal swab for HPV. Specimen (iii) and residual material from specimens (i) and (ii) were tested for type-specific HPV DNA. HPV detection was by an in-house multiplex PCR and Luminex-based genotyping assay.

Results

A total of 129 MSM were recruited of mean age 38.1 years; 24\% were HIV-positive. Of these 92/129 (71\%) had any type-specific HPV DNA in $\geq 1$ specimen; 80/129 (62\%) had HR-HPV.

Of 123 participants with sufficient residual pooled and dedicated specimens, 70 (56.9\%) had detectable HPV on both and 40 (32.5\%) were negative on both; overall concordance $89 \%(95 \% \mathrm{Cl}$ 83\%,94\%), kappa statistic $0.78(0.66,0.89)$. Pooled samples had a 4.1\% (95\% Cl -1.9\%,10.0\%) higher test positive rate than dedicated samples.

Of 125 participants with sufficient residual rectal and specimens, 74 (59.2\%) had detectable HPV on both and 36 (28.8\%) were negative on both; overall concordance $88 \%(95 \% \mathrm{Cl} 81 \%, 93 \%)$, kappa statistic $0.74(95 \% \mathrm{Cl} 0.61,0.86)$. Residual rectal samples had 5.6\% $(95 \% \mathrm{Cl}-0.6 \%, 11.8 \%)$ higher test positivity than dedicated samples.

\section{Conclusions}

We observed high concordance between the dedicated and residual STI test specimens. Our data support the strategy of testing residual specimens for HPV prevalence monitoring in MSM to evaluate the impact of the targeted vaccination programme.

\section{Manuscript}

Word count $=2999$

\section{Background}

Gay, bisexual and other men who have sex with men (MSM) are at high risk of sexually transmitted infection with high risk (HR) oncogenic strains of human papillomavirus (HPV) and HPV-related neoplasia, in particular anal cancer.(1) Infection with the most common HR-HPV genotypes is 
vaccine preventable. HPV vaccination was introduced for adolescent females in the UK in 2008 to protect against HPV-related cervical cancer. Initially the programme used the bivalent vaccine Cervarix which targets HR-HPV genotypes 16 and 18, responsible for most cervical cancers.(2) Since 2012 the quadrivalent vaccine Gardasil ${ }^{\circledR}$ has been offered which targets the same genotypes, 16 and 18 , as well as low risk genotypes 6 and 11 which cause anogenital warts. A nonavalent vaccine targeting five additional HR genotypes was licensed in June 2015 but is not currently used in the national vaccine programme in the UK. Adolescent males became eligible for vaccination with quadrivalent vaccine in September 2019.

Following the introduction of vaccination in adolescent females, marked declines in HPV infection and HPV-related disease have been observed in young adult women and heterosexual men but not MSM, who do not benefit from the indirect protective effect of immunity in the vaccinated female population.(3-6) To address the persistent high rates of HPV-related morbidity in MSM, a programme of targeted, opportunistic HPV vaccination was introduced for MSM aged $\leq 45$ years attending sexual health services (SHS) in England using the quadrivalent vaccine (3 dose schedule). The programme, coordinated by Public Health England (PHE), was introduced as a pilot at 42 selected SHS sites from April 2016 and extended to all SHS in England from April 2018.(7)

Screening for sexually transmitted infections (STI) in MSM typically includes collection of swab specimens from the rectum and oropharynx and a first void urine (FVU) specimen for nucleic acid amplification testing (NAAT) for Chlamydia trachomatis (CT) and Neisseria Gonorrhoeae (NG).(8) Pooling of specimens, whereby the FVU, oropharyngeal and rectal specimens are combined into a single specimen is employed by some SHS to reduce testing costs.(9) HPV DNA is detectable on anal swab specimens from MSM with current anal HPV infection. Plans for monitoring the impact of the targeted vaccination programme include regular surveillance of anal HPV prevalence in MSM attending SHS. The collection procedure for an anal swab specimen for HPV testing and for rectal CT/NG NAAT are similar. PHE have proposed that residual material from rectal CT/NG NAAT specimens collected from MSM attending SHS could be tested for HPV DNA to monitor the prevalence in this population.(10) However, HPV detection in residual rectal or pooled NAAT specimens in men has not previously been evaluated. 
We designed a cross-sectional study to compare HPV DNA detection in residual rectal and pooled samples taken for CT/NG NAAT with a dedicated anal swab sample for HPV DNA testing. The study was designed as a pilot to trial the study processes and provide an updated estimate of anal HPV prevalence in MSM.

\section{Methods}

Study design

The study was conducted at a large SHS in central London with a high proportion of attendees who are gay or bisexual men. Eligible participants were self-identified MSM aged $\geq 16$ years attending for asymptomatic STI screening or care relating to genital symptoms.

Participant eligibility was confirmed at the routine consultation by the attending clinician who then obtained written consent. All participants provided:

(i) a pooled oropharyngeal/rectal/FVU specimen for CT/NG NAAT

(ii) a rectal swab specimen for CT/NG NAAT

(iii) a dedicated anal swab specimen for HPV testing

Participants would ordinarily give either (i) or (ii), but not both, at routine sexual healthcare visits. Pooled specimens are taken as standard from most asymptomatic MSM attendees; symptomatic individuals, or those reporting contact with a confirmed case of CT/NG infection, provide separate FVU, oropharyngeal and rectal specimens.

\section{Specimen collection}

The materials and collection procedure for the NAAT specimens were those used for standard clinical care at the study centre. Pooled specimens comprised of an oropharyngeal swab (Copan ${ }^{\circledR}$ plastic flocked swab, Copan Diagnostics, Murrieta, CA.) rubbed against the posterior pharynx. The swab was agitated inside a Hologic ${ }^{\circledR}$ Aptima ${ }^{\circledR}$ (Hologic, Inc, Marlborough, MA) urine specimen tube containing $2.0 \mathrm{ml}$ of transport medium and then discarded. A rectal swab (Copan ${ }^{\circledR}$ plastic flocked swab) was subsequently inserted 2-3cm inside the anal canal and rotated for 5-10 seconds, then placed inside the same specimen tube. The FVU sample was collected by the patient in a separate universal container and returned to clinic staff who decanted $2 \mathrm{ml}$ of urine into the tube to form a 
pooled oropharyngeal/rectal/FVU specimen. The rectal NAAT specimen employed a Hologic ${ }^{\circledR}$ Aptima ${ }^{\circledR}$ cotton-tip swab inserted $2-3 \mathrm{~cm}$ inside the anal canal, rotated for 5-10 seconds, then placed inside a specimen tube containing $2.9 \mathrm{ml}$ Aptima ${ }^{\circledR}$ transport medium

The dedicated anal HPV swab was a Copan ${ }^{\circledR}$ flocked swab, pre-soaked in sterile saline, inserted $3 \mathrm{~cm}$ into the anal canal and rotated 360 degrees, a standard method described in a prior HPV study at the same centre.(11) The swab was subsequently placed inside a tube containing Copan ${ }^{\circledR}$ universal transport medium. To align with routine clinical pathways at the study site, all three specimens could be self-taken by asymptomatic participants or clinician-taken for symptomatic participants. All participants and clinicians received written instructions for sample collection.

To eliminate any potential systematic impact of prior swabbing on HPV detection in any one specimen type, study ID numbers were allocated in equal numbers to one of the six possible orders of the three anorectal swabs and the collection order specified within the instructions for each participant ID.

Specimen transport

The pooled or rectal NAAT specimen required for routine care was sent for CT/NG testing according to usual clinic procedures. These were maintained at room temperature in the $\mathrm{CT} / \mathrm{GC}$ testing laboratory. Once CT/NG testing was complete (within 7 days) the residual samples were retained in the $\mathrm{CT} / \mathrm{NG}$ testing laboratory at room temperature for $\leq 7$ additional days before transfer to the HPV testing laboratory at PHE. The additional NAAT specimens and dedicated HPV specimens were refrigerated at $4^{\circ} \mathrm{C}$ at the study site and stored for $\leq 14$ days before transport to the HPV testing laboratory. Duplicate CT/NG testing on the additional NAAT specimen was not performed.

\section{Laboratory methods}

All specimens were processed within 7 days of arrival at the HPV testing laboratory and $300 \mu \mathrm{l}$ aliquots were stored at $-25^{\circ} \mathrm{C}$. HPV testing on all specimens was carried out in June 2018 using a non-commercial assay employed extensively in prior UK HPV prevalence studies (see online supplement for further details).(11-13) The assay incorporates a universal probe which detects all mucosal HPV genotypes and differential detection of 21 individual HPV genotypes. Of these, genotypes 16/18/31/33/35/39/45/51/52/56/58/59/68 were considered HR-HPV, genotypes 6/11 were considered low-risk (LR) and genotypes 26/53/66/70/73/82 were considered possible HR-HPV 
in accordance with currently accepted categorisation.(14) Specimen integrity was established by PCR targeting the human pyruvate dehydrogenase $(\mathrm{PDH})$ gene to indicate sufficient cellular material. Specimens in which neither HPV nor PDH DNA was detected were considered inadequate.

Statistical methods

Prevalence was measured for any genotype-specific HPV (any of 21 genotypes detected by the assay), HR-HPV, quadrivalent HPV (qHPV) and nonavalent HPV (9vHPV) (any of the 4 and 9 genotypes covered by quadrivalent and nonavalent vaccination respectively). HPV prevalence was estimated by HIV status and prior GHPV vaccine exposure and differences evaluated by the $\mathrm{X}^{2}$ test. Prevalence of any genotype-specific HPV and qHPV were measured by specimen type and the difference evaluated by McNemar's exact test. Confidence intervals $(\mathrm{Cl})$ around estimates of the difference in test positive rates for any genotype-specific and qHPV for each specimen type were determined at $95 \%$ using standard methods for binomial Cls for a proportion.(15) Kappa statistics were calculated for each comparison with 95\% confidence intervals using a recommended method. $(16,17)$ Statistical analyses were conducted using STATA v15 (StataCorp LP, College Station, TX, USA).

Sample size

A sample size of 119 was required to achieve an estimated 62 true-positive cases of any genotypespecific HPV; further details of the power calculation appear in the supplementary material.

Results

\section{Participant characteristics}

A total of 129 participants were recruited in February - April 2018 (see Table 1). Mean age was 38.1 years (IQR 31-46 years). 31/129 (24\%) were HIV positive. 65 (50.4\%) had never had HPV vaccination; 15 (11.6\%) had received 1 dose of qHPV vaccine prior to their attendance; 14 (10.9\%) had 2 prior doses and 35 (27.1\%) had completed 3 doses of vaccine. 
Specimen management

Specimen flow is shown in Figure 1. Of 387 specimens collected, 367 (94.8\%) were adequate for HPV detection. All 129 participants had $\geq 1$ adequate specimen.

HPV Prevalence

The prevalence of $\geq 1$ type-specific HPV genotype was $92 / 129$ participants (71\%). Prevalence by individual genotype is shown in Figure 2. Prevalence was $49 / 129$ (38.0\%) for $\geq 1$ qHPV vaccine genotype, 76/129 (58.9\%) for $\geq 1$ 9vHPV genotype and 80/129 (62.0\%) for $\geq 1$ HR-HPV type. Infection with multiple ( $\geq 2$ ) genotypes was detected in 68 participants $(52.7 \%)$.

Prevalence of $\geq 1$ type-specific HPV infection was $27 / 31$ (87.1\%) in HIV-positive participants and 65/98 (66.3\%) HIV-negative participants, $P=0.026$. For $\geq 1$ qHPV genotype, prevalence was 16/31 (51.6\%) and 33/98 (33.7\%) in HIV positive and negative participants respectively, $\mathrm{P}=0.07$.

Type-specific HPV infection was present in 43/65 (66.2\%) in those with no prior HPV vaccination; $29 / 35(82.9 \%)$ in those who had completed 3 doses of vaccine and 11/15 (73.3\%) and 9/14 (64.3\%) in those with 1 and 2 previous doses respectively, $P=0.32$. Prevalence of $\geq 1 \mathrm{qHPV}$ vaccine genotype was $24 / 65$ (36.9\%) in those with no prior HPV vaccination; $15 / 35$ (42.9\%) in those who had completed vaccination and 6/15 (40.0\%) and 4/14 (28.6\%) in those with 1 and 2 previous doses, $\mathrm{P}=0.82$.

Specimen concordance

Paired concordance data by HPV categorisation is summarised in Table 2; frequency of discordant results by individual genotype is shown in supplementary Table S1.

Dedicated vs residual pooled specimens

123 participants had an adequate dedicated and pooled specimen. Pooled samples had $4.1 \%(95 \% \mathrm{Cl}$ $-1.9,10.0)$ higher test positivity than dedicated samples for any type-specific HPV and $0.8 \%(95 \% \mathrm{Cl}$ $-4.2,5.9)$ higher for qHPV. 
70/123 participants (56.9\%) had detectable type-specific HPV DNA on both specimens and 40 (32.5\%) were negative on both (total $89.4 \%$ concordant). 9/123 (7.3\%) had a positive pooled but negative dedicated specimen, and 4/123 (3.3\%) had a positive dedicated but negative pooled specimen (total $10.6 \%$ discordant); $\mathrm{P}=0.17$, kappa 0.78 (95\% Cl 0.66, 0.89).

Dedicated vs residual rectal specimen

125 participants had an adequate dedicated and rectal specimen. Rectal samples had $5.6 \%(95 \% \mathrm{Cl}$ $-0.6,11.8)$ higher test positivity than dedicated samples for any type-specific HPV and $2.4 \%(95 \% \mathrm{Cl}$ $-2.0,6.8$ ) higher for qHPV.

74/125 participants (59.2\%) had detectable type-specific HPV DNA on both specimens and 36/125 (28.8\%) were negative on both (total $88 \%$ concordant). $11 / 125$ (8.8\%) had a positive rectal but negative dedicated specimen, and 4/125 (3.2\%) had a positive dedicated but negative rectal specimen (total $12 \%$ discordant); $\mathrm{P}=0.07$, kappa 0.74 (95\% $\mathrm{Cl} 0.61,0.86)$.

Residual rectal vs pooled specimen

119 participants had an adequate rectal and pooled specimen. Rectal samples had $1.7 \%(95 \% \mathrm{Cl}-3.3$, 6.6) higher test positivity than pooled samples for any type-specific HPV and $0.8 \%(95 \% \mathrm{Cl}-4.4,6.0)$ for qHPV.

74/119 participants (62.2\%) had detectable type-specific HPV DNA on both specimens and 37/119 (31.1\%) were negative on both (total 93.3\% concordant). 3/119 (2.5\%) had a positive pooled but negative rectal specimen and 5/119 $(4.2 \%)$ had a positive rectal but negative pooled specimen (total $6.7 \%$ discordant); kappa 0.85 (95\% $\mathrm{Cl} 0.75,0.95)$.

Sample integrity

Quantitative glyceraldehyde 3-phosphate dehydrogenase (GAPDH) PCR was performed on a subset of study participants ( $\mathrm{n}=44 ; 34 \%$ of total) to estimate the number of cells collected per $\mathrm{mL}$ of sample. The geometric means $(95 \% \mathrm{Cl})$ for the estimate number of cells per $\mathrm{mL}$ of sample were as indicated: 
dedicated anal swab 11,819 (4,831 - 28,916); residual pooled specimen 27,991 (11,208 - 69,907; $\mathrm{p}=0.07)$ and residual rectal specimen $114,643(60,056-218,846 ; p<0.001)$.

Table 1: Participant characteristics $(n=129)$

\begin{tabular}{|c|c|c|}
\hline Age (years) & $\begin{array}{l}\text { Mean: } 38.1 \\
\text { Median: } 38\end{array}$ & IQR: 31-46 \\
\hline & $\mathrm{n}$ & $\%$ \\
\hline \multicolumn{3}{|l|}{ HIV status } \\
\hline Positive & 31 & 24.0 \\
\hline Negative & 98 & 76.0 \\
\hline \multicolumn{3}{|l|}{ Smoking history } \\
\hline Current smoker & 24 & 18.6 \\
\hline Ex-smoker & 25 & 19.4 \\
\hline Lifelong non-smoker & 80 & 62.0 \\
\hline \multicolumn{3}{|l|}{ Sexual behaviour } \\
\hline Receptive and insertive anal intercourse & 72 & 55.8 \\
\hline Receptive anal intercourse only & 21 & 16.3 \\
\hline Insertive anal intercourse only & 26 & 20.2 \\
\hline No anal intercourse & 10 & 7.8 \\
\hline \multicolumn{3}{|l|}{ History of anogenital warts } \\
\hline Current warts & 3 & 2.3 \\
\hline Previous warts & 31 & 19.4 \\
\hline No previous/current warts & 95 & 62.0 \\
\hline \multicolumn{3}{|l|}{ Genital symptoms* } \\
\hline Urethral & 8 & 6.2 \\
\hline Rectal & 1 & 0.7 \\
\hline Other** & 5 & 3.1 \\
\hline None & 116 & 89.9 \\
\hline \multicolumn{3}{|l|}{ Concurrent bacterial STI } \\
\hline Any & 24 & 18.6 \\
\hline Chlamydia & 7 & 5.4 \\
\hline Gonorrhoea & 16 & 12.4 \\
\hline Syphilis & 3 & 2.3 \\
\hline \multicolumn{3}{|l|}{ Specimen-taker } \\
\hline Self-performed & 119 & 92.2 \\
\hline Clinician-performed & 10 & 7.8 \\
\hline \multicolumn{3}{|l|}{ Prior quadrivalent HPV vaccination } \\
\hline $\mathrm{Nil}$ & 65 & 50.4 \\
\hline 1 dose & 15 & 11.6 \\
\hline 2 doses & 14 & 10.9 \\
\hline 3 doses & 35 & 27.1 \\
\hline
\end{tabular}

Footnote to Table 1

* Participants may be included in $>1$ category if they have multiple symptoms

** Included testicular pain, oropharyngeal ulceration, anogenital warts, Pthirus Pubis and penile glans rash 
Table 2: Specimen concordance

\begin{tabular}{|c|c|c|c|c|c|c|}
\hline & \multicolumn{6}{|c|}{ Dedicated $(D)$ vs residual pooled $(P)$ specimens $(n=123)$} \\
\hline & $D+P+$ & D - P - & $D+P-$ & D - P + & $\begin{array}{l}\text { Concordance } \\
\text { (\%) }\end{array}$ & $\begin{array}{l}\text { Kappa } \\
(95 \% \mathrm{Cl}) \\
\end{array}$ \\
\hline $\begin{array}{l}\text { Any } \\
\text { genotyped } \\
\text { HPV }\end{array}$ & 70 & 40 & 4 & 9 & 89.4 & $\begin{array}{l}0.78 \\
(0.66,0.89)\end{array}$ \\
\hline qHPV & 35 & 79 & 4 & 5 & 92.7 & $\begin{array}{l}0.83 \\
(0.73,0.94)\end{array}$ \\
\hline 9vHPV & 53 & 53 & 6 & 11 & 86.2 & $\begin{array}{l}0.72 \\
(0.60,0.85)\end{array}$ \\
\hline HR-HPV & 56 & 52 & 6 & 9 & 87.8 & $\begin{array}{l}0.76 \\
(0.64,0.87) \\
\end{array}$ \\
\hline \multirow[t]{3}{*}{ HPV 16/18 } & 19 & 98 & 2 & 4 & 95.1 & $\begin{array}{l}0.83 \\
(0.71,0.96) \\
\end{array}$ \\
\hline & \multicolumn{6}{|c|}{ Dedicated (D) vs residual rectal (R) specimens $(n=125)$} \\
\hline & $\mathrm{D}+\mathrm{R}+$ & $\mathbf{D}-\mathbf{R}-$ & D+R - & D - R+ & & \\
\hline $\begin{array}{l}\text { Any } \\
\text { genotyped } \\
\text { HPV }\end{array}$ & 74 & 36 & 4 & 11 & 88.0 & $\begin{array}{l}0.74 \\
(0.61,0.86)\end{array}$ \\
\hline qHPV & 38 & 80 & 2 & 5 & 94.4 & $\begin{array}{l}0.87 \\
(0.78,0.96)\end{array}$ \\
\hline 9vHPV & 57 & 55 & 5 & 8 & 89.6 & $\begin{array}{l}0.74 \\
(0.62,0.85)\end{array}$ \\
\hline HR-HPV & 57 & 47 & 8 & 13 & 83.2 & $\begin{array}{l}0.66 \\
(0.53,0.79)\end{array}$ \\
\hline \multirow[t]{3}{*}{ HPV 16/18 } & 18 & 100 & 3 & 4 & 94.4 & $\begin{array}{l}0.80 \\
(0.66,0.94)\end{array}$ \\
\hline & \multicolumn{6}{|c|}{ Residual rectal (R) vs residual pooled (P) specimens ( $n=119)$} \\
\hline & $R+P+$ & $\mathbf{R}-\mathbf{P}$ - & $\mathbf{R}+\mathbf{P}-$ & $\mathbf{R}-\mathbf{P}+$ & & \\
\hline $\begin{array}{l}\text { Any } \\
\text { genotyped } \\
\text { HPV }\end{array}$ & 74 & 37 & 5 & 3 & 93.3 & $\begin{array}{l}0.85 \\
(0.75,0.95)\end{array}$ \\
\hline qHPV & 36 & 74 & 5 & 4 & 92.4 & $\begin{array}{l}0.83 \\
(0.73,0.94) \\
\end{array}$ \\
\hline $9 \mathrm{vHPV}$ & 56 & 50 & 5 & 8 & 89.1 & $\begin{array}{l}0.78 \\
(0.67,0.89) \\
\end{array}$ \\
\hline HR-HPV & 60 & 50 & 5 & 4 & 92.4 & $\begin{array}{l}0.85 \\
(0.75,0.94)\end{array}$ \\
\hline HPV 16/18 & 17 & 93 & 3 & 6 & 92.4 & $\begin{array}{l}0.75 \\
(0.59,0.90) \\
\end{array}$ \\
\hline
\end{tabular}


Discussion

This is the first study to evaluate HPV detection in residual STI specimens in men against dedicated HPV specimens of the sort commonly employed in prevalence studies. We observed high concordance between all specimens without any suggestion of lower HPV detection in either residual specimen type. Even if the true detection were at the lower limit of the $95 \% \mathrm{Cl}$ for residual rectal specimens ( $0.6 \%$ lower for genotype-specific HPV, $2.0 \%$ lower for qHPV) and residual pooled specimens (2.9\% lower for genotype-specific HPV, 4.2\% lower for qHPV) compared to dedicated specimens, this margin of error is likely acceptable for epidemiological surveillance. We concluded that our pilot data provided sufficient evidence for the study objective without progressing to the larger study initially proposed.

Whilst we observed high concordance between all three specimen types for all clinically relevant categories of HPV, concordance was consistently greater for qHPV than for any genotype-specific HPV. This likely reflects the reduced potential for variability when comparing the smaller number of genotypes tested per participant. The high concordance between residual rectal and pooled specimens is reassuring, such that any shift in STI testing practice towards increased use of pooling of specimens is unlikely to compromise prevalence measurements in future surveillance.

Several factors may have contributed to the discordance we observed. Small differences in results will arise between any serially performed specimens. The material differences between the three specimen types, including swab type (flocked Copan ${ }^{\circledR}$ swab for the pooled NAAT and dedicated specimens versus cotton-tip Hologic ${ }^{\circledR}$ swab for rectal NAAT specimens) and pre-soaking in saline of the dedicated swab specimen may have impacted the amount of cellular material collected. This observation is supported by our additional assessment of sample integrity in which cell counts estimated through GAPDH quantification were greater for both residual specimens. Whist our study cannot fully isolate the impact of each variable, our results suggest that the cotton swab is at least as effective for HPV sampling and that pre-soaking of anal swabs may be unnecessary or even reduce the quantity of cellular material collected. Multiple studies have found detectable HPV in oropharyngeal and urine specimens in MSM, albeit with a lower prevalence than in anal specimens. $(11,18,19)$ A small proportion of the HPV DNA found in our pooled samples may therefore originate from the FVU and oropharyngeal components of the specimen.

We chose to express our results in terms of concordance rather than sensitivity and specificity. Whilst the methodology employed for the dedicated HPV specimens is commonplace for anal HPV 
prevalence studies, evidence is lacking that it constitutes a true "gold standard" for HPV detection relative to other proposed sampling methods. We hypothesize that the discordance seen between specimens arose from the factors outlined above, rather than constituting false positive and false negative results in the residual specimens. Although the confidence intervals for the difference between them cross zero, our observation of marginally higher HPV detection in the residual specimens suggests that they performed as well or slightly better than the dedicated specimen, rather than having lower specificity.

The proportion of inadequate specimens, defined by the absence of HPV or PDH DNA, was low for all three specimen types. This suggests that the use of residual NAAT material, or pooling with oropharyngeal and FVU samples does not negatively impact the integrity of cellular DNA. . The majority of specimens on our study were self-taken by participants. Whilst we acknowledge that it is not a controlled evaluation of self versus clinician sampling, our findings nonetheless suggest that self-taken samples are adequate for monitoring HPV prevalence.

Our prevalence estimates were similar to those recorded in a prevalence study amongst MSM attending the same clinical site 6 years previously (see supplementary material).(20) Anal HPV was significantly more prevalent in MSM living with HIV, an established independent risk factor for HPV infection.(1) Our cohort included some participants who had commenced or completed qHPV vaccination from the pilot opportunistic programme during the 2 years preceding study participation. Whilst we observed similar levels of qHPV in our vaccinated and unvaccinated participants, the qHPV DNA detected in vaccinated participants may have constituted persistent infection acquired prior to vaccination, re-infection, reactivation of latent infection or deposition following recent sexual activity. The cross-sectional design of our study cannot differentiate these possibilities and does not permit evaluation of the effect of vaccine on any of these scenarios. Whilst the efficacy of vaccine in preventing de novo HPV infection is clearly established(21, 22), recent vaccine exposure will not impact persistent HPV infection acquired prior to vaccination.

Specimens taken for routine STI testing were stored at room temperature by the STI testing laboratory whilst the duplicate NAAT study specimens were refrigerated at $4^{\circ} \mathrm{C}$. We acknowledge that the duplicate NAAT specimens should ideally have matched the storage conditions of the STI test specimens but we feel that the difference is unlikely to have impacted our results. The product literature for the specimen collection kits for both specimens recommend storage between $2-30^{\circ} \mathrm{C}$ and the proportion of inadequate specimens with absent cellular DNA was low for both groups of specimens. $(23,24)$ 
Our sample of MSM were at high risk of STI acquisition and may not reflect MSM attending sexual health services nationally, or the UK MSM population in general. However, the objective of the study was to compare the performance of HPV detection from different sampling methods therefore recruitment of a study population with a high number of HPV positive samples was desirable. It is possible that any serially performed anal swab specimens would exhibit a similar degree of discordance to that observed. The inclusion of an additional duplicate dedicated specimen would have allowed us to evaluate this but risked additional inconvenience to participants, potential for error in sample management and increased testing costs.

Our data support using residual STI screening specimens to monitor HPV prevalence in MSM and surveillance data based on such specimens have been reported.(25) Their use facilitates HPV surveillance without disruption to routine sexual healthcare practices and without the collection of additional specimens. We also observed high concordance between residual rectal and pooled samples. This finding provides reassurance that should care pathways evolve to expand pooling of STI test specimens, testing of residual pooled specimens can provide comparable estimates of HPV prevalence for the purposes of surveillance.

\section{Figure 1: Sample management from collection through to testing}

Figure 2: Type-specific prevalence by genotype

\section{Acknowledgements}

We are very grateful to the study participants and thank them for their time and effort; this research would not have been possible without their contribution which we value greatly. We thank Dr Jaime Vera, Brighton \& Sussex University Hospital for providing external peer review of the study protocol. We would also like to thank the research nurses and doctors at the UCL Centre for Clinical Research in Infection and Sexual Health and the clinical staff at the Mortimer Market Centre for their assistance in participant recruitment and specimen collection. Many thanks also to the STI testing laboratory staff at TDL Pathology for their assistance in retaining and transporting the residual specimens.

Funding

HPV testing costs were funded by Pubic Health England (Vaccines and Counter Measures Budget). 
Ethical approval

The study received ethical approval from the East Midlands/Leicester Central Research Ethics Committee (REC) on $7^{\text {th }}$ December 2017 (REC reference 17/EM/0473) and NHS Health Research Authority (HRA) approval on $2^{\text {nd }}$ January 2018 (Integrated Research Application System (IRAS) project ID 234139). All study participants provided informed, written consent.

Author contribution

RG, KS and DM conceived the idea for the study. RG and DN developed the study design. DN developed the study protocol, prepared the study materials, led participant recruitment and wrote the study manuscript. OS provided statistical guidance for the study design, sample size calculation and analysis of results. KP and SB carried out HPV testing of study specimens. RG, SP, OS, KP, DM, MC, KS and SB advised on the study design and reviewed and edited the manuscript.

1. Machalek DA, Poynten M, Jin F, Fairley CK, Farnsworth A, Garland SM, et al. Anal human papillomavirus infection and associated neoplastic lesions in men who have sex with men: A systematic review and meta-analysis. The Lancet Oncology. 2012;13(5):487-500.

2. Mesher D, Cuschieri K, Hibbitts S, Jamison J, Sargent A, Pollock KG, et al. Type-specific HPV prevalence in invasive cervical cancer in the UK prior to national HPV immunisation programme: baseline for monitoring the effects of immunisation. J Clin Pathol. 2015;68(2):135-40.

3. Mesher D, Panwar K, Thomas SL, Edmundson C, Choi YH, Beddows S, et al. The Impact of the National HPV Vaccination Program in England Using the Bivalent HPV Vaccine: Surveillance of TypeSpecific HPV in Young Females, 2010-2016. J Infect Dis. 2018;218(6):911-21.

4. Drolet M, Benard E, Perez N, Brisson M, Ali H, Boily MC, et al. Population-level impact and herd effects following the introduction of human papillomavirus vaccination programmes: updated systematic review and meta-analysis. The Lancet. 2019;394(10197):497-509.

5. Chow EP, Read TR, Wigan R, Donovan B, Chen MY, Bradshaw CS, et al. Ongoing decline in genital warts among young heterosexuals 7 years after the Australian human papillomavirus (HPV) vaccination programme. Sex Transm Infect. 2015;91(3):214-9.

6. Public Health England. Sexually transmitted infections and screening for chlamydia in England, 2018: Health Protection Report Volume 13 Number 19; published 4th June 2019; available at:

https://assets.publishing.service.gov.uk/government/uploads/system/uploads/attachment data/file 806118/hpr1919 stis-ncsp ann18.pdf; accessed 14th February 2020.

7. Edelstein M, Iyanger N, Hennessy N, Mesher D, Checchi M, Soldan K, et al. Implementation and evaluation of the human papillomavirus (HPV) vaccination pilot for men who have sex with men (MSM), England, April 2016 to March 2017. Euro Surveill. 2019;24(8). 
8. Clutterbuck D, Asboe D, Barber T, Emerson C, Field N, Gibson S, et al. 2016 United Kingdom national guideline on the sexual health care of men who have sex with men. Int J STD AIDS.

2018:956462417746897.

9. Sultan B, White JA, Fish R, Carrick G, Brima N, Copas A, et al. The "3 in 1" Study: Pooling SelfTaken Pharyngeal, Urethral, and Rectal Samples into a Single Sample for Analysis for Detection of Neisseria gonorrhoeae and Chlamydia trachomatis in Men Who Have Sex with Men. J Clin Microbiol. 2016;54(3):650-6.

10. Checchi M, Mesher D, McCall M, Coukan F, Chau C, Mohammed H, et al. HPV vaccination of gay, bisexual and other men who have sex with men in sexual health and HIV clinics in England: vaccination uptake and attendances during the pilot phase. Sex Transm Infect. 2019;95(8):608-13.

11. King EM, Gilson R, Beddows S, Soldan K, Panwar K, Young C, et al. Human papillomavirus DNA in men who have sex with men: type-specific prevalence, risk factors and implications for vaccination strategies. Br J Cancer. 2015;112(9):1585-93.

12. Bissett SL, Howell-Jones R, Swift C, De Silva N, Biscornet L, Parry JV, et al. Human papillomavirus genotype detection and viral load in paired genital and urine samples from both females and males. J Med Virol. 2011;83(10):1744-51.

13. Johnson AM, Mercer CH, Beddows S, de Silva N, Desai S, Howell-Jones R, et al. Epidemiology of, and behavioural risk factors for, sexually transmitted human papillomavirus infection in men and women in Britain. Sex Transm Infect. 2012;88(3):212-7.

14. Bouvard V, Baan R, Straif K, Grosse Y, Secretan B, El Ghissassi F, et al. A review of human carcinogens--Part B: biological agents. Lancet Oncol. 2009;10(4):321-2.

15. Newcombe RG. Improved confidence intervals for the difference between binomial proportions based on paired data. Stat Med. 1998;17(22):2635-50.

16. Landis JR, Koch GG. The measurement of observer agreement for categorical data. Biometrics. 1977;33(1):159-74.

17. Fleiss JL, Levin BA, Paik MC. Statistical methods for rates and proportions. 3rd ed. / Joseph L. Fleiss, Bruce Levin, Myunghee Cho Paik. ed. Hoboken, N.J. ; [Chichester]: Wiley-Interscience; 2003.

18. Giuliano AR, Nielson CM, Flores R, Dunne EF, Abrahamsen M, Papenfuss MR, et al. The optimal anatomic sites for sampling heterosexual men for human papillomavirus (HPV) detection: the HPV detection in men study. J Infect Dis. 2007;196(8):1146-52.

19. King EM, Oomeer S, Gilson R, Copas A, Beddows S, Soldan K, et al. Oral human papillomavirus infection in men who have sex with men: A systematic review and meta-analysis. PLoS One. 2016;11(7).

20. King EM. Human papillomavirus epidemiology in men who have sex with men: implications for a vaccine programme at sexual health clinics in the UK: University College London; 2015.

21. Palefsky JM, Giuliano AR, Goldstone S, Moreira ED, Jr., Aranda C, Jessen H, et al. HPV vaccine against anal HPV infection and anal intraepithelial neoplasia. N Engl J Med. 2011;365(17):1576-85.

22. Giuliano AR, Palefsky JM, Goldstone S, Moreira Jr ED, Penny ME, Aranda C, et al. Efficacy of quadrivalent $\mathrm{HPV}$ vaccine against HPV infection and disease in males. $\mathrm{N}$ Engl $\mathrm{J}$ Med. 2011;364(5):401-11.

23. Hologic Inc. Aptima Urine Specimen Collection Kit for Male and Female Urine Specimens; available at: https://www.hologic.com/sites/default/files/package-insert/502260-IFU-

PI 002 01.pdf; accessed 14th February 2020.

24. Hologic Inc. Aptima Unisex Swab Specimen Collection Kit for Endocervical and Male Urethral Swab Specimens; available at: https://www.hologic.com/sites/default/files/2019-06/502258-IFUPI 003 01.pdf; accessed 14th February 2020.

25. Cameron RL, Cuschieri K, Pollock KGJ. Baseline HPV prevalence in rectal swabs from men attending a sexual health clinic in Scotland: assessing the potential impact of a selective HPV vaccination programme for men who have sex with men. Sex Transm Infect. 2020;96(1):55-7. 


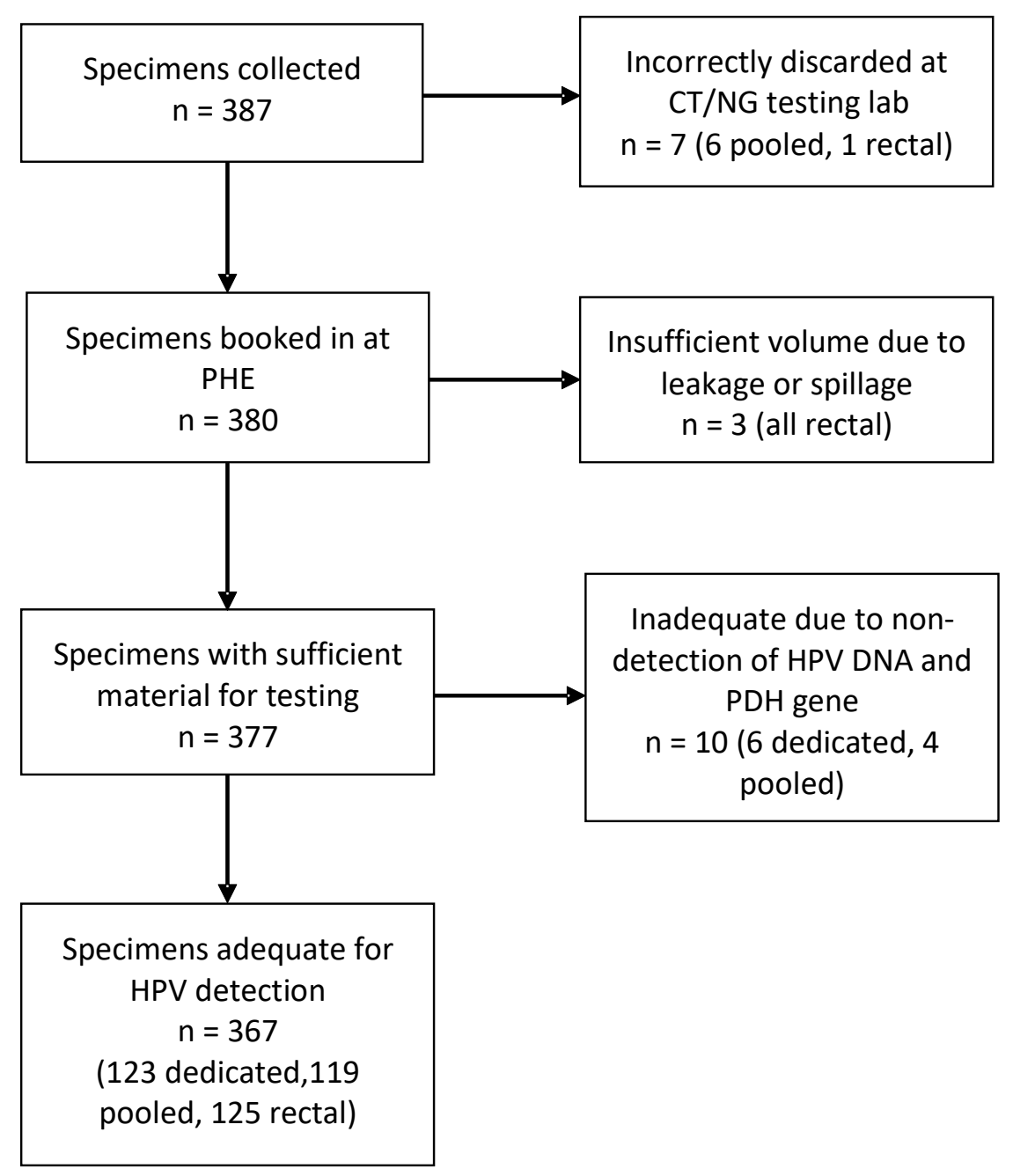




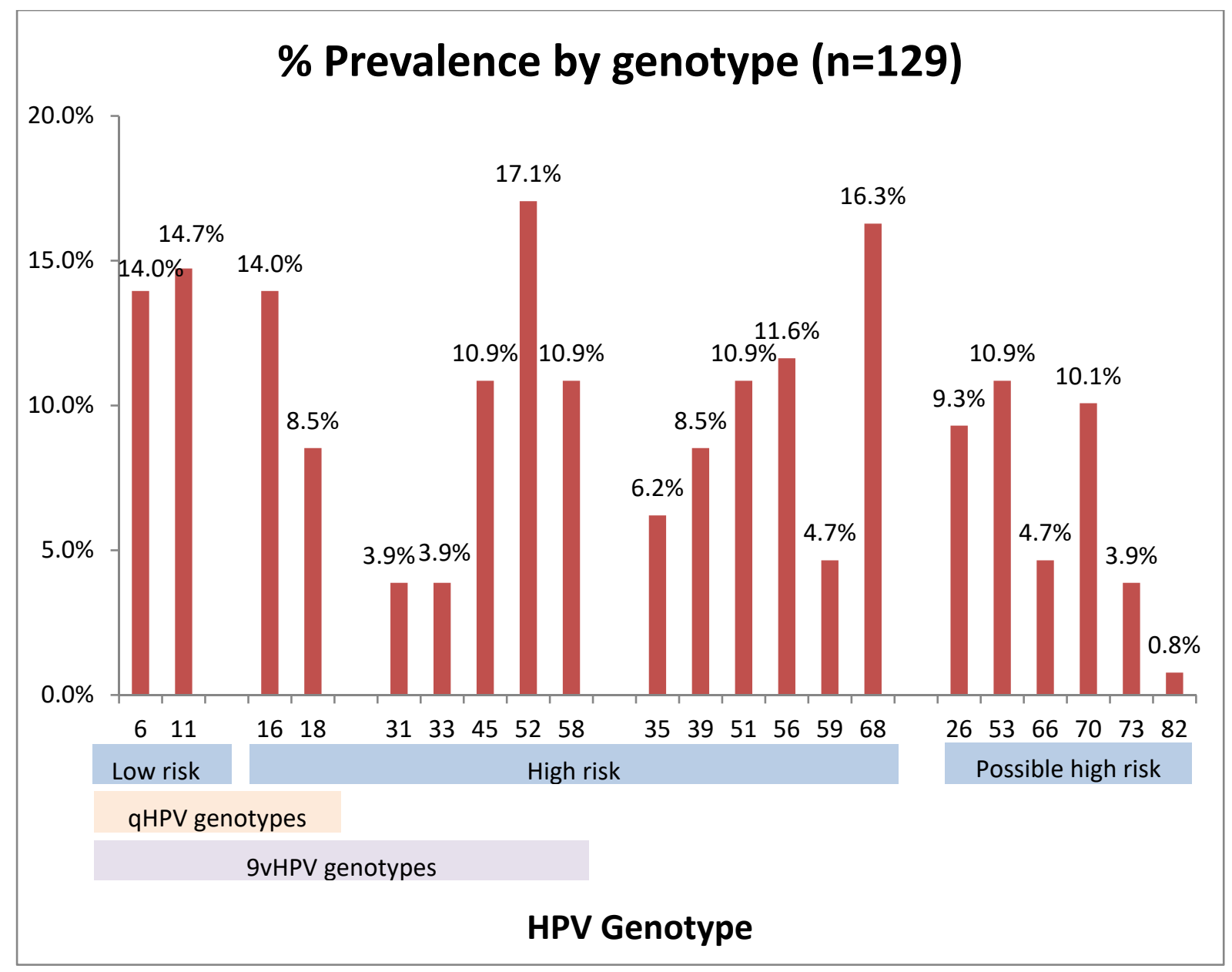

